ESAIM: M2AN 48 (2014) 1757-1775

DOI: $10.1051 / \mathrm{m} 2 \mathrm{an} / 2014018$
ESAIM: Mathematical Modelling and Numerical Analysis

www.esaim-m2an.org

\title{
REGULARITY AND APPROXIMABILITY OF THE SOLUTIONS TO THE CHEMICAL MASTER EQUATION*
}

\author{
Ludwig Gauckler $^{1}$ And Harry Yserentant ${ }^{1}$
}

\begin{abstract}
The chemical master equation is a fundamental equation in chemical kinetics. It underlies the classical reaction-rate equations and takes stochastic effects into account. In this paper we give a simple argument showing that the solutions of a large class of chemical master equations are bounded in weighted $\ell_{1}$-spaces and possess high-order moments. This class includes all equations in which no reactions between two or more already present molecules and further external reactants occur that add mass to the system. As an illustration for the implications of this kind of regularity, we analyze the effect of truncating the state space. This leads to an error analysis for the finite state projections of the chemical master equation, an approximation that forms the basis of many numerical methods.
\end{abstract}

Mathematics Subject Classification. 80A30, 60J27, 65L05, 65L70.

Received May 9, 2013. Revised January 23, 2014.

Published online October 3, 2014.

\section{INTRODUCTION}

The chemical master equation is a fundamental equation in chemical kinetics. It underlies the classical reaction-rate equations and takes the stochastic effects into account that cannot be neglected in the case of small population numbers. Such situations often arise in biological systems. See the recent reviews $[6,11,12]$ and the classical papers $[4,5]$ for an introduction. There is an ongoing effort to tackle the chemical master equation numerically, the major challenge being its high dimensionality: for a system of $d$ interacting species the chemical master equation is a differential equation with state space $\mathbb{N}_{0}^{d}, \mathbb{N}_{0}$ the set of nonnegative integers. Various numerical methods have been proposed, for instance Galerkin methods [1], spectral methods [3], sparse grid methods [7,9], wavelet methods [15,24], tensor methods $[2,8,14,16]$, and hybrid methods $[9,10,13,19]$. In the papers on numerical methods known to us, no attention is paid to the regularity (or the decay) of the solutions to the chemical master equation, although such properties are inherently related to their approximability and are particularly relevant in high dimensions. In the present work, we give a simple argument showing high regularity in weighted $\ell_{1}$-spaces for a large class of chemical master equations, including all those in which no reactions between two or more molecules and further external reactants occur that add mass to the system. Almost all examples considered in the aforementioned papers are of this kind. As an illustration for the implications of

Keywords and phrases. Chemical master equation, existence of solutions and moments, error of finite state projections.

* This research was supported by the DFG-Research Center MATHEon.

1 Institut für Mathematik, Technische Universität Berlin, Straße des 17. Juni 136, 10623 Berlin, Germany.

gauckler@math.tu-berlin.de; yserentant@math.tu-berlin.de 
this kind of regularity we analyze the effect of truncating the state space. This leads to an error analysis for the finite state projection $[20,22]$ of the chemical master equation, an approximation that is more or less explicitly behind most numerical methods and forms in some sense their basis.

The chemical master equation can be regarded as a differential-difference equation, a differential equation in time and a difference equation in the rest of the variables, or in other words a possibly infinite system

$$
\frac{\mathrm{d}}{\mathrm{d} t} p(\Omega ; t, x)=\sum_{r=1}^{R}\left(a_{r}\left(t, x-\nu_{r}\right) p\left(\Omega ; t, x-\nu_{r}\right)-a_{r}(t, x) p(\Omega ; t, x)\right), \quad x \in \Omega,
$$

of ordinary differential equations. The solution components are labeled by vectors $x \in \mathbb{Z}^{d}$ and take the value

$$
p(\Omega ; t, x)=0, \quad x \notin \Omega,
$$

for all $x$ outside a given subset $\Omega$ of $\mathbb{Z}^{d}$. The $\nu_{r}$ are given elements in $\mathbb{Z}^{d}$ and the coefficient functions

$$
a_{r}: \mathbb{R}_{\geq 0} \times \mathbb{Z}^{d} \rightarrow \mathbb{R}_{\geq 0}:(t, x) \rightarrow a_{r}(t, x)
$$

are assumed to be nonnegative and continuous in the variable $t$ for each single $x$. We denote their sum by

$$
a(t, x)=\sum_{r=1}^{R} a_{r}(t, x) .
$$

The differential equations (1.1) relate the functions $t \rightarrow p(\Omega ; t, x), x \in \Omega$, to at most $R$ of their neighbors in this set and form a local balance law. This fixes the notion of solution and means the system (1.1) has to be understood in naive sense, as a collection of possibly infinitely many ordinary differential equations. The remaining solution parts (1.2) are mainly introduced by formal reasons. They serve to simplify the notation and to obtain a joint domain of definition when relating solutions on different sets $\Omega$ to each other.

In chemical kinetics, $\Omega$ is a subset of $\mathbb{N}_{0}^{d}$ or in the limit case the set $\mathbb{N}_{0}^{d}$ itself. The value $p(\Omega ; t, x)$ represents the probability that there are $x_{i}$ copies of the molecule $\mathrm{S}_{\mathrm{i}}$ at time $t$. Consider an elementary reaction $r$,

$$
\mathrm{m}_{1} \mathrm{~S}_{1}+\ldots+\mathrm{m}_{\mathrm{d}} \mathrm{S}_{\mathrm{d}} \longrightarrow \mathrm{n}_{1} \mathrm{~S}_{1}+\ldots+\mathrm{n}_{\mathrm{d}} \mathrm{S}_{\mathrm{d}}
$$

in which $m_{1}$ molecules of species $1, m_{2}$ molecules of species $2, \ldots$ react to $n_{1}$ molecules of species $1, n_{2}$ molecules of species 2 and so on. The corresponding vector $\nu_{r}$, the stoichiometric vector of the reaction, is composed of the differences $n_{i}-m_{i}$. The coefficient function $a_{r}(t, x)$, that is denoted as propensity function of the reaction, is in chemical kinetics a constant or time-dependent multiple of the polynomial

$$
a_{r}(t, x) \sim \prod_{i=1}^{d} \frac{x_{i} !}{\left(x_{i}-m_{i}\right) !}
$$

of order $m_{1}+\ldots+m_{d}$ in the components $x_{1}, x_{2}, \ldots, x_{d}$ of $x$ as long as $x_{i} \geq m_{i}$ for all $i$ and takes the value 0 otherwise. That is, it is proportional to the number of possibilities to select the reacting molecules from the given ones. The here omitted factor of proportionality measures the reactivity of the substances and can depend on time, for example via external influences like temperature.

If the set $\Omega$ is finite, (1.1) is a linear system of ordinary differential equations of usual kind for the solution components $p(\Omega ; t, x), x \in \Omega$. Existence and uniqueness of its solution for given initial values $p(\Omega ; 0, x), x \in \Omega$, is guaranteed by standard results from the theory of ordinary differential equations. The situation is different for infinite sets $\Omega$, and in particular for the limit case

$$
\Omega=\mathbb{N}_{0}^{d}
$$


in which one is primarily interested. The aim of this note is to study the minimal nonnegative solutions of the chemical master equation (1.1) on arbitrary subsets $\Omega$ of $\mathbb{Z}^{d}$ for nonnegative, summable initial values, solutions that are the pointwise minima of all possible such solutions and uniquely determined by this property.

The existence of such solutions can be shown by an elegant, comparatively elementary argument by Reuter and Ledermann [21]. Martcheva et al. [18] use semigroup techniques to study a related class of infinite systems of ordinary differential equations in $\ell_{1}$-like sequence spaces. The present paper is strongly influenced by the work of Reuter and Ledermann and utilizes the techniques developed there. Our primary aim is to show that the solutions constructed following Reuter and Ledermann grow at most like

$$
\sum_{x \in \Omega} \gamma(t, x) p(\Omega ; t, x) \leq c(t) \sum_{x \in \Omega} \gamma(0, x) p(\Omega ; 0, x)
$$

where $\gamma: \mathbb{R}_{\geq 0} \times \mathbb{Z}^{d} \rightarrow \mathbb{R}_{\geq 0}$ is a weight function that is differentiable in $t$ and satisfies an estimate

$$
\dot{\gamma}(t, x)+\sum_{r=1}^{R} a_{r}(t, x)\left(\gamma\left(t, x+\nu_{r}\right)-\gamma(t, x)\right) \leq \kappa(t) \gamma(t, x)
$$

for all $x$ in the domains $\Omega$ under consideration, where $\kappa: \mathbb{R}_{\geq 0} \rightarrow \mathbb{R}_{\geq 0}$ is a given continuous function and

$$
c(t)=\exp \left(\int_{0}^{t} \kappa(s) \mathrm{d} s\right)
$$

As we will show, estimates like (1.8) have striking consequences for the regularity of the solutions both with respect to the discrete variables and with respect to time.

We will restrict ourselves in the discussion of this condition to subsets $\Omega$ of $\mathbb{N}_{0}^{d}$ and weight functions not depending on time. The trivial example of such a weight function satisfying an estimate $(1.9)$ is $\gamma(t, x)=1$. In this case, $\kappa=0$ and (1.8) transfers to the estimate

$$
\sum_{x \in \Omega} p(\Omega ; t, x) \leq \sum_{x \in \Omega} p(\Omega ; 0, x)
$$

The other extreme is exponentially growing weight functions like

$$
\gamma(t, x)=\exp (\vartheta\langle x\rangle), \quad\langle x\rangle=\sum_{i=1}^{d} x_{i} M_{i},
$$

with $\vartheta>0$ and $M_{1}, \ldots, M_{d}>0$ given parameters, in chemical kinetics the molecular masses of the species. An estimate (1.9) then holds if the coefficient functions $a_{r}(t, x)$ are bounded for those $r$ for which $\left\langle\nu_{r}\right\rangle>0$; the behavior of the other coefficient functions is of no significance in this context.

In view of applications to chemical kinetics, we are primarily interested in polynomial weight functions. The key observation is that the differences $\gamma\left(t, x+\nu_{r}\right)-\gamma(t, x)$ are then polynomials of one order lower degree than the degree of the weight functions themselves. We will consider polynomial-like weight functions

$$
\gamma(t, x)=\max (1,\langle x\rangle)^{m}
$$

where the exponent $m$ can be an arbitrary real number greater than zero. The crucial estimate (1.9) is then satisfied if the coefficient functions $a_{r}(t, x)$ grow at most linearly in $x$, or more precisely in the given weigthed sum of the components of $x$, for all those reactions $r$ for which $\left\langle\nu_{r}\right\rangle>0$, that is, if for these $r$

$$
a_{r}(t, x) \leq \alpha(t)\langle x\rangle
$$


for all $x \neq 0$ in $\Omega$, respectively $\mathbb{N}_{0}^{d}$ with some continuous function $\alpha$. In chemical kinetics, this excludes only elementary reactions between two or more already present molecules and further external reactants that add mass to the system. If the single reactions conserve mass, the weighted sums $\left\langle\nu_{r}\right\rangle$ of the components of their assigned stoichiometric vectors $\nu_{r}$ take the value zero. The condition (1.9) then holds with $\kappa(t)=0$, not only for the weight functions (1.13) but for all weight functions $\gamma$ depending only on the total mass $\langle x\rangle$.

The rest of this article is organized as follows. In Section 2 we study the case of finite state spaces $\Omega$ using the fact that the chemical master equation reduces in this case to a finite set of ordinary differential equations. In particular, we show that the solutions remain nonnegative for nonnegative initial values and satisfy the basic estimate (1.8). In Section 3 we show that the solutions increase when enlarging the domain $\Omega$. Together with the results from Section 2, this observation forms the basis of our proof of the existence of minimal solutions in Section 4, which is adapted from the work [21] of Reuter and Ledermann. It is then easy to transfer the stability estimate (1.8) to the case of possibly infinite state spaces $\Omega$. We show that it implies in situations as present in chemical kinetics a high-order regularity in the space-like variables $x$ and in time and reinterpret these results in a functional-analytic setting. In Section 5, we show by examples that the basic estimate (1.8) can in general not be improved and that one cannot do without growth conditions to the coefficient functions like (1.9). This is not truly surprising as the chemical master equation can be used to describe rather violent chain reactions. In Section 6, our stability estimates are reinterpreted in terms of the discrete Fourier transform of the solutions. It turns out that the corresponding Fourier series represent, under given conditions, infinitely differentiable functions of the frequency variables. Since smooth periodic functions can be approximated in many different ways, observations of this kind may be helpful in the construction and analysis of numerical methods. In Section 7, we identify properties that guarantee that a solution remains a probability distribution when time evolves. These conditions are fulfilled in the case of the coefficient functions (1.6) and fundamental for the probabilistic interpretation of the chemical master equation. The last section is finally devoted to an error analysis for the finite state projections of the solutions on the basis of our stability result. We show, roughly speaking, that one reaches an arbitrarily high convergence order for corresponding initial values.

\section{The Chemical Master Equation on Finite state SPACES}

We begin studying the case of finite subsets $\Omega$ of $\mathbb{N}_{0}^{d}$ or more generally of $\mathbb{Z}^{d}$. As mentioned, the existence and uniqueness of the solutions of the chemical master equation (1.1), (1.2) for given initial values is then guaranteed by standard results from the theory of ordinary differential equations and does not pose a problem. For the rest of this section, we will fix the state space $\Omega$ and will denote the solutions by

$$
p(t, x)=p(\Omega ; t, x) .
$$

Our first observation is the well-known fact that the solutions remain nonnegative for nonnegative initial values.

Lemma 2.1. If the set $\Omega$ is finite and $p(0, x) \geq 0$ for all $x \in \Omega$, the solution $p(t, x)=p(\Omega ; t, x)$ of the chemical master equation (1.1), (1.2) remains nonnegative for all $t \geq 0$.

Proof. Let $T>0$ be arbitrarily given. Since the coefficient functions $a_{r}(t, x)$ are continuous in $t$ for any $x$, and since $\Omega$ contains only finitely many $x$, there exists a constant $\mu \geq 0$ such that $a(t, x) \leq \mu$ holds for the sum (1.4) of the coefficient functions for $0 \leq t \leq T$ and all $x \in \Omega$. First we study the exponentially weighted functions $q(t, x)=\mathrm{e}^{\mu t} p(t, x)$ on the interval $0 \leq t \leq T$. They satisfy the differential equations

$$
\frac{\mathrm{d}}{\mathrm{d} t} q(t, x)=\mu q(t, x)+\sum_{r=1}^{R}\left(a_{r}\left(t, x-\nu_{r}\right) q\left(t, x-\nu_{r}\right)-a_{r}(t, x) q(t, x)\right), \quad x \in \Omega,
$$

which are, in obvious vector notation, equivalent to a system

$$
q(t)=q(0)+\int_{0}^{t}(\mu I+A(s)) q(s) \mathrm{d} s, \quad 0 \leq t \leq T,
$$


of integral equations that can be solved by Picard-iteration. Since the integral operator on the right hand side maps, by the choice of the constant $\mu$, componentwise nonnegative functions to componentwise nonnegative functions, the solution of this integral equation is componentwise nonnegative for $0 \leq t \leq T$. Thus

$$
p(t, x) \geq 0, \quad x \in \Omega,
$$

for $0 \leq t \leq T$, and, since $T>0$ was arbitrary, for all $t \geq 0$.

We come now to our central estimate (1.8), here first for solutions on finite state spaces $\Omega$.

Lemma 2.2. If the set $\Omega$ is finite and $p(0, x) \geq 0$ for all $x \in \Omega$, the then nonnegative solution $p(t, x)=p(\Omega ; t, x)$ of the chemical master equation (1.1), (1.2) satisfies the a priori estimate

$$
\sum_{x \in \Omega} \gamma(t, x) p(t, x) \leq c(t) \sum_{x \in \Omega} \gamma(0, x) p(0, x)
$$

where $\gamma(t, x)$ is a weight function that is assumed to satisfy the estimate (1.9) and $c(t)$ the function (1.10).

Proof. To simplify matters, we restrict ourselves first to the case that the weight function $\gamma$ does not depend on $t$. Since the sum is finite, we can differentiate the left hand side of (2.2) and obtain from (1.1)

$$
\frac{\mathrm{d}}{\mathrm{d} t} \sum_{x \in \Omega} \gamma(x) p(t, x)=\sum_{r=1}^{R} \sum_{x \in \Omega}\left(a_{r}\left(t, x-\nu_{r}\right) \gamma(x) p\left(t, x-\nu_{r}\right)-a_{r}(t, x) \gamma\left(x+\nu_{r}\right) p(t, x)\right)+\Delta(t),
$$

where we have used the abbreviation

$$
\Delta(t)=\sum_{r=1}^{R} \sum_{x \in \Omega} a_{r}(t, x)\left(\gamma\left(x+\nu_{r}\right)-\gamma(x)\right) p(t, x)
$$

Because of the nonnegativity of all terms and since $p(t, x)=0$ for all $x$ outside $\Omega$,

$$
\sum_{x \in \Omega} a_{r}\left(t, x-\nu_{r}\right) \gamma(x) p\left(t, x-\nu_{r}\right) \leq \sum_{x \in \Omega} a_{r}(t, x) \gamma\left(x+\nu_{r}\right) p(t, x),
$$

as the sum on the left hand side is a sum of function values at points in a subset of $\Omega$. The double sum on the right hand side of the differential equation above hence attains a value less than or equal to zero, which leads to

$$
\frac{\mathrm{d}}{\mathrm{d} t} \sum_{x \in \Omega} \gamma(x) p(t, x) \leq \sum_{r=1}^{R} \sum_{x \in \Omega} a_{r}(t, x)\left(\gamma\left(x+\nu_{r}\right)-\gamma(x)\right) p(t, x)
$$

and, as $p(t, x)$ is nonnegative, by using (1.9) finally to the estimate

$$
\frac{\mathrm{d}}{\mathrm{d} t} \sum_{x \in \Omega} \gamma(x) p(t, x) \leq \kappa(t) \sum_{x \in \Omega} \gamma(x) p(t, x) .
$$

The estimate (2.2) now follows by means of Gronwall's lemma. If $\gamma$ depends on $t$, the additional term

$$
\sum_{x \in \Omega} \dot{\gamma}(t, x) p(t, x)
$$

arises, that is covered by assumption (1.9), too. 
The lemma applies in particular to the weight function $\gamma=1$, for which $\kappa=0$, and leads to the basic estimate

$$
\sum_{x \in \Omega} p(t, x) \leq \sum_{x \in \Omega} p(0, x)
$$

for the nonnegative solutions of the chemical master equation already mentioned in the introduction.

The question arises whether an exponential growth as in (2.2) can actually be observed. The answer is no, not for finite state spaces and time-independent weight functions. The reason is obvious. As follows from (2.3),

$$
\sum_{x \in \Omega} \gamma(x) p(t, x) \leq\left(\max _{x \in \Omega} \gamma(x)\right) \sum_{x \in \Omega} p(0, x)
$$

That means that the left hand side of this inequality remains bounded for all times $t \geq 0$. The point is, however, that we need bounds that are independent of $\Omega$ and still hold when $\Omega$ approaches an infinite domain like $\mathbb{N}_{0}^{d}$.

\section{The MONOTONICITY PRINCIPLE}

Our aim is to extend the results of Section 2 from finite to infinite state spaces $\Omega$, and in particular to the state space $\Omega=\mathbb{N}_{0}^{d}$. Following Reuter and Ledermann [21], we start from the following monotonicity principle.

Lemma 3.1. Let $\Omega$ be an arbitrary and $\Omega^{\prime} \subseteq \Omega$ be a finite subset of $\mathbb{Z}^{d}$ and let $p\left(\Omega^{\prime} ; t, x\right)$ and $p(\Omega ; t, x)$ be nonnegative solutions of the chemical master equation (1.1), (1.2) on the state spaces $\Omega^{\prime}$ and $\Omega$, respectively. Then

$$
p\left(\Omega^{\prime} ; t, x\right) \leq p(\Omega ; t, x)
$$

for all $x \in \mathbb{Z}^{d}$ and all $t \geq 0$, provided this holds at the initial time $t=0$.

Proof. The proof is based on the same idea as that of Lemma 2.1. Let $T>0$ be arbitrarily given and let $\mu$ be an upper bound for the sum (1.4) of the coefficient functions for $0 \leq t \leq T$ and $x$ in the finite set $\Omega^{\prime}$. Let

$$
e(t, x)=\mathrm{e}^{\mu t} \chi(x)\left\{p(\Omega ; t, x)-p\left(\Omega^{\prime} ; t, x\right)\right\},
$$

with $\chi$ the characteristic function of the finite set $\Omega^{\prime}$. Moreover, let

$$
\sigma(t, x)=\mathrm{e}^{\mu t} \sum_{r=1}^{R}\left(1-\chi\left(x-\nu_{r}\right)\right) a_{r}\left(t, x-\nu_{r}\right) p\left(\Omega ; t, x-\nu_{r}\right) .
$$

For all elements $x$ in the finite set $\Omega^{\prime}$ then

$$
\frac{\mathrm{d}}{\mathrm{d} t} e(t, x)=\mu e(t, x)+\sum_{r=1}^{R}\left(a_{r}\left(t, x-\nu_{r}\right) e\left(t, x-\nu_{r}\right)-a_{r}(t, x) e(t, x)\right)+\sigma(t, x) .
$$

As in the proof of Lemma 2.1, one deduces from that that the functions $t \rightarrow e(t, x), x \in \Omega^{\prime}$, considered as the unique solution of the above system of differential equations with given right hand side $\sigma(t, x) \geq 0$ and given initial values $e(0, x) \geq 0$, are nonnegative for $0 \leq t \leq T$. As $T>0$ was arbitrary and $p\left(\Omega^{\prime} ; t, x\right)$ vanishes outside the set $\Omega^{\prime}$, this proves the inequality (3.1). 


\section{EXISTEnCE AND REgUlarity of Minimal SOLUtions}

With the help of the technique of Reuter and Ledermann [21], we are now in the position to treat the case of the initial value problem (1.1), (1.2) on arbitrary subsets $\Omega$ of $\mathbb{Z}^{d}$, in particular on the set $\Omega=\mathbb{N}_{0}^{d}$. We start from a summable set of nonnegative initial values $p(0, x), x \in \Omega$, and first introduce the finite state projections

$$
p\left(\Omega^{\prime} ; t, x\right), \quad \Omega^{\prime} \text { a finite subset of } \Omega,
$$

the solutions of the chemical master equation (1.1), (1.2) on the sets $\Omega^{\prime}$ for the initial values

$$
p\left(\Omega^{\prime} ; 0, x\right)=p(0, x), \quad x \in \Omega^{\prime} .
$$

Theorem 4.1. Let $\Omega_{1} \subseteq \Omega_{2} \subseteq \ldots$ be a sequence of finite subsets of $\mathbb{Z}^{d}$ that exhaust $\Omega$ and $p(0, x), x \in \Omega$, be a summable set of nonnegative initial values. The corresponding finite state projections $p\left(\Omega_{n} ; t, x\right), n=1,2,3, \ldots$, then converge pointwise in $t$ and $x$ monotonously from below to a nonnegative solution $p(t, x)=p(\Omega ; t, x)$ of the chemical master equation on the set $\Omega$ for the given initial values that satisfies, for all $t \geq 0$, the estimate

$$
\sum_{x \in \Omega} p(t, x) \leq \sum_{x \in \Omega} p(0, x)
$$

The nonzero components $t \rightarrow p(t, x), x \in \Omega$, of this solution are continuously differentiable functions of $t$.

Proof. The finite state projections $p\left(\Omega_{n} ; t, x\right)$ are by Lemma 2.1 nonnegative and satisfy by $(2.3)$ the estimate

$$
\sum_{x \in \Omega} p\left(\Omega_{n} ; t, x\right) \leq \sum_{x \in \Omega} p(0, x)
$$

For each single $x \in \mathbb{Z}^{d}$, the functions $t \rightarrow p\left(\Omega_{n} ; t, x\right)$ converge therefore by Lemma 3.1 monotonously from below pointwise to a measurable, nonnegative limit function $t \rightarrow p(t, x)$. The sum of these limit functions satisfies, for every $t \geq 0$, the estimate (4.3). The functions $t \rightarrow p\left(\Omega_{n} ; t, x\right), x \in \Omega_{n}$, satisfy the differential equations

$$
\frac{\mathrm{d}}{\mathrm{d} t} p\left(\Omega_{n} ; t, x\right)=\sum_{r=1}^{R}\left(a_{r}\left(t, x-\nu_{r}\right) p\left(\Omega_{n} ; t, x-\nu_{r}\right)-a_{r}(t, x) p\left(\Omega_{n} ; t, x\right)\right),
$$

which can be rewritten as integral equations

$$
p\left(\Omega_{n} ; t, x\right)=p\left(\Omega_{n} ; 0, x\right)+\int_{0}^{t} \sum_{r=1}^{R}\left(a_{r}\left(s, x-\nu_{r}\right) p\left(\Omega_{n} ; s, x-\nu_{r}\right)-a_{r}(s, x) p\left(\Omega_{n} ; s, x\right)\right) \mathrm{d} s .
$$

These integral equations are in particular fulfilled for all $x$ in a fixed set $\Omega_{m}$ and all indices $n$ greater than or equal to the given $m$. The integrands are by the estimate above, and because of the continuity of the coefficient functions in the time variable, bounded on the interval $0 \leq s \leq t$ by constants that can depend on $t$ and $x$ but are independent of $n$. The dominated convergence theorem leads therefore to the integral equations

$$
p(t, x)=p(0, x)+\int_{0}^{t} \sum_{r=1}^{R}\left(a_{r}\left(s, x-\nu_{r}\right) p\left(s, x-\nu_{r}\right)-a_{r}(s, x) p(s, x)\right) \mathrm{d} s,
$$

first only for the elements $x$ in the set $\Omega_{m}$ under consideration. But as the index $m$ was chosen arbitrarily and the subsets $\Omega_{m}$ exhaust $\Omega$, the limit functions $t \rightarrow p(t, x)$ satisfy these integral equations for all $x \in \Omega$. As these limit functions and the coefficient functions as well are locally bounded in the time variable, the integrands are locally bounded and the limit functions $t \rightarrow p(t, x)$ therefore continuous. As the coefficient functions and with 
that also the integrands are continuous in the integration variable, the limit functions $t \rightarrow p(t, x)$ are thus, by the fundamental theorem of calculus, continuously differentiable. They satisfy the differential equations

$$
\frac{\mathrm{d}}{\mathrm{d} t} p(t, x)=\sum_{r=1}^{R}\left(a_{r}\left(t, x-\nu_{r}\right) p\left(t, x-\nu_{r}\right)-a_{r}(t, x) p(t, x)\right)
$$

for all $x \in \Omega$ and take the given initial values, that is, they form a solution of the chemical master equation.

Theorem 4.1 implies, for fixed $t \geq 0$, the convergence of the finite state projections $p\left(\Omega_{n} ; t, x\right)$ in the $\ell_{1}$-sense to the constructed solution $p(t, x)=p(\Omega ; t, x)$ of the initial value problem. That is, for all fixed $t \geq 0$

$$
\lim _{n \rightarrow \infty} \sum_{x \in \Omega}\left|p(\Omega ; t, x)-p\left(\Omega_{n} ; t, x\right)\right|=0 .
$$

The reason is that the summands are bounded by the summable function $x \rightarrow p(\Omega ; t, x)$ and tend pointwise to zero as $n$ goes to infinity. The proposition thus follows from the dominated convergence theorem in its version for absolutely summable series. The locally uniform convergence in time will be studied later.

The solutions from Theorem 4.1 are characterized by two extremal properties. Let $\Omega^{\prime}$ be an arbitrary finite subset of the set $\Omega$ under consideration. Since the $\Omega_{n}$ exhaust $\Omega, \Omega^{\prime}$ is a subset of $\Omega_{n}$ for all sufficiently large indices $n$. By the monotonicity principle from Lemma 3.1 for these $n$

$$
p\left(\Omega^{\prime} ; t, x\right) \leq p\left(\Omega_{n} ; t, x\right) \leq p(\Omega ; t, x) .
$$

On the other hand since $p\left(\Omega_{n} ; t, x\right)$ tends to the value $p(\Omega ; t, x)$ of the constructed solution as $n$ goes to infinity, this observation shows that this solution is the least upper bound of the finite state projections. Moreover,

$$
p\left(\Omega^{\prime} ; t, x\right) \leq \widetilde{p}(\Omega ; t, x)
$$

for every finite state projection $p\left(\Omega^{\prime} ; t, x\right)$ and any other nonnegative solution $\widetilde{p}(\Omega ; t, x)$ attaining the same initial values as $p(\Omega ; t, x)$, which follows again from Lemma 3.1. We summarize:

Theorem 4.2. For summable nonnegative initial values $p(\Omega ; 0, x)$, the then finite least upper bound

$$
p(\Omega ; t, x)=\sup \left\{p\left(\Omega^{\prime} ; t, x\right) \mid \Omega^{\prime} \subseteq \Omega \text { finite, } p\left(\Omega^{\prime} ; 0, x\right)=p(\Omega ; 0, x) \text { for } x \in \Omega^{\prime}\right\}
$$

of the finite state projections $p\left(\Omega^{\prime} ; t, x\right)$ solves the initial value problem (1.1), (1.2) on the set $\Omega$ under consideration and satisfies the estimate (4.3). This solution is at the same time the minimum of all possible nonnegative solutions of the system (1.1) for the given initial values.

The results from [17] show that such initial value problems can possess more than one solution. The minimum solution from Theorem 4.2 is, however, unique by definition. Theorem 4.2 thus offers a selection principle for the physically meaningful solutions, similar to the entropy principle in the study of hyperbolic conservation laws. If the set $\Omega$ is finite, the least upper bound (4.7) of the finite state projections is the finite state projection $p(\Omega ; t, x)$, that is, as expected, the unique solution of the then finite system (1.1), (1.2).

For summable, not necessarily nonnegative initial values $p(0, x)$, one can compose from the given minimal solutions $p_{-}$and $p_{+}$for the nonnegative initial values

$$
p_{-}(0, x)=\max (0,-p(0, x)), \quad p_{+}(0, x)=\max (0, p(0, x)) .
$$

a solution of the chemical master equation on the set $\Omega$ under consideration, namely

$$
p(t, x)=p_{+}(t, x)-p_{-}(t, x) .
$$

For finite state spaces $\Omega$, the so defined solution is, of course, again the unique solution of the finite system (1.1), (1.2) of ordinary differential equations for the given initial values. 
Theorem 4.3. The solutions (4.9) depend linearly on the initial values and satisfy the estimate

$$
\sum_{x \in \Omega}|p(t, x)| \leq \sum_{x \in \Omega}|p(0, x)|
$$

That is, the chemical master equation is in this sense $\ell_{1}$-contractive.

Proof. As the finite state projections depend linearly on the initial data, so do the solutions (4.9). The solution parts $p_{-}$and $p_{+}$are nonnegative and can therefore be estimated with help of Theorem 4.1. Because

$$
p_{-}(0, x)+p_{+}(0, x)=|p(0, x)|
$$

the proposition thus follows applying the triangle inequality to the left hand side of (4.10).

We will restrict ourselves in the sequel to the minimum nonnegative solutions from Theorem 4.2 respectively to the solutions (4.9) of arbitrary sign composed of them. It is then easy to see that the a priori estimate from Lemma 2.2 transfers to the present case of possibly infinite sets $\Omega$ and in particular to the set $\Omega=\mathbb{N}_{0}^{d}$.

Theorem 4.4. For initial values for which the sum on the right hand side of the following equation attains a finite value, the solutions of the chemical master equation considered in Theorem 4.3 satisfy the estimate

$$
\sum_{x \in \Omega} \gamma(t, x)|p(t, x)| \leq c(t) \sum_{x \in \Omega} \gamma(0, x)|p(0, x)|
$$

where $\gamma(t, x)$ is a weight function that is assumed to satisfy the estimate (1.9) and $c(t)$ the function (1.10).

Proof. We restrict ourselves at first to nonnegative solutions. Lemma 2.2 yields in this case

$$
\sum_{x \in \Omega^{\prime}} \gamma(t, x) p\left(\Omega_{n} ; t, x\right) \leq c(t) \sum_{x \in \Omega} \gamma(0, x) p(0, x)
$$

for every sequence of finite sets $\Omega_{n}$ that exhaust $\Omega$ and every given finite subset $\Omega^{\prime}$ of $\Omega$. As $n$ goes to infinity,

$$
\sum_{x \in \Omega^{\prime}} \gamma(t, x) p(t, x) \leq c(t) \sum_{x \in \Omega} \gamma(0, x) p(0, x)
$$

follows, and from that, if one lets tend $\Omega^{\prime}$ to $\Omega$, finally the estimate (4.11) for the case of nonnegative initial values. This estimate can again be applied separately to the two parts of a solution (4.9), from which the proposition follows by the same argument as in the proof of the previous theorem.

In view of applications in chemical kinetics, we are mainly interested in polynomial-like weight functions, in particular in the weight function (1.13), and may assume that $\Omega$ is a subset of the $d$-fold cartesian product $\mathbb{N}_{0}^{d}$ of the set of the nonnegative integers. The crucial condition (1.9) is in this case satisfied if the coefficient functions $a_{r}(t, x)$ for which $\left\langle\nu_{r}\right\rangle>0$ grow at most linearly in $x$ in the sense of (1.14).

Theorem 4.5. Let $\Omega$ be a subset of $\mathbb{N}_{0}^{d}$ and let the coefficient functions $a_{r}(t, x)$ for which $\left\langle\nu_{r}\right\rangle>0$ grow at most linearly in $x$ in the sense of (1.14). For any given exponent $m>0$, the weighted $\ell_{1}$-norms

$$
|p(t, 0)|+\sum_{x \in \Omega}\langle x\rangle^{m}|p(t, x)|
$$

of the solutions (4.9) remain then uniformly bounded on finite time intervals if they are finite at time $t=0$. 
The discrete "spatial" regularity of the solutions expressed by Theorem 4.5 translates directly to estimates for their time derivatives. For ease of presentation, we restrict ourselves in the sequel to the case of coefficient functions not depending on time, that is, to chemical master equations

$$
\frac{\mathrm{d}}{\mathrm{d} t} p(t, x)=\sum_{r=1}^{R}\left(a_{r}\left(x-\nu_{r}\right) p\left(t, x-\nu_{r}\right)-a_{r}(x) p(t, x)\right)
$$

on the domain $\Omega=\mathbb{N}_{0}^{d}$. The equation (4.13) relates the continuous time derivative of every single solution component $t \rightarrow p(t, x), x \in \Omega$, to finitely many other such components. These components are therefore infinitely differentiable functions of time and their time derivatives of given order are linear combinations of finitely many other such components in a given fixed, finite neighborhood pattern. This proves

Theorem 4.6. Assume that the coefficient functions of the equation (4.13) grow at most polynomially in $x$, and those for which $\left\langle\nu_{r}\right\rangle>0$ at most linearly in $x$ in the sense of (1.14). If the norms (4.12) of a solution (4.9) of this equation are then finite at time $t=0$ and with that by Theorem 4.5 at all times $t \geq 0$, the single components $t \rightarrow p(t, x), x \in \Omega$, of this solution are infinitely differentiable functions of $t$ and the expressions

$$
\sum_{x \in \Omega}\langle x\rangle^{m}\left|\frac{\mathrm{d}^{k}}{\mathrm{~d} t^{k}} p(t, x)\right|
$$

remain uniformly bounded on finite time intervals for all differentiation orders $k$ and all exponents $m \geq 0$.

Theorem 4.6 can be reinterpreted in the language of functional analysis, considering the solutions as mappings from the positive real axis to the space $\ell_{1}$ over the set $\Omega=\mathbb{N}_{0}^{d}$ or its polynomially weighted counterparts.

Theorem 4.7. Let the expressions (4.14) be uniformly bounded on finite time intervals. The function

$$
\mathbb{R}_{\geq 0} \rightarrow \ell_{1}\left(\mathbb{N}_{0}^{d}\right): t \rightarrow p(t, \cdot)
$$

is then infinitely differentiable. The components of its $k$ th derivative are the $k$ th derivatives

$$
\frac{\mathrm{d}^{k}}{\mathrm{~d} t^{k}} p(t, x)
$$

of its components. The same holds in the weighted $\ell_{1}$-spaces with the polynomial-like weights (1.13).

Proof. We introduce the abbreviation $p_{k}(t, x)$ for the $k$ th derivative of the component $t \rightarrow p(t, x)$ and fix at first an arbitrary time $T>0$. For any given finite subset $\Omega^{\prime}$ of $\mathbb{N}_{0}^{d}$ and all $t_{0}<t \leq T$ then

$$
\sum_{x \in \Omega^{\prime}}\left|p_{k}(t, x)-\left\{p_{k}\left(t_{0}, x\right)+p_{k+1}\left(t_{0}, x\right)\left(t-t_{0}\right)\right\}\right| \leq \int_{t_{0}}^{t}(t-s) \sum_{x \in \Omega^{\prime}}\left|p_{k+2}(s, x)\right| \mathrm{d} s
$$

holds, which results from the Taylor expansion

$$
f(t)=f\left(t_{0}\right)+f^{\prime}\left(t_{0}\right)\left(t-t_{0}\right)+\int_{t_{0}}^{t}(t-s) f^{\prime \prime}(s) \mathrm{d} s
$$

of twice continuously differentiable functions, here applied to the corresponding components. By assumption, the sum under the integral on the right hand side in the estimate above is bounded by a constant $C_{k}(T)$ that is independent of the choice of the finite set $\Omega^{\prime}$. This leads to the estimate

$$
\sum_{x \in \Omega^{\prime}}\left|p_{k}(t, x)-\left\{p_{k}\left(t_{0}, x\right)+p_{k+1}\left(t_{0}, x\right)\left(t-t_{0}\right)\right\}\right| \leq \frac{1}{2} C_{k}(T)\left(t-t_{0}\right)^{2},
$$


which obviously also holds if $t<t_{0}$. Letting $\Omega^{\prime}$ tend to $\Omega=\mathbb{N}_{0}^{d}$, this finally yields the $\ell_{1}$-norm estimate

$$
\left\|p_{k}(t, \cdot)-\left\{p_{k}\left(t_{0}, \cdot\right)+p_{k+1}\left(t_{0}, \cdot\right)\left(t-t_{0}\right)\right\}\right\| \leq \frac{1}{2} C_{k}(T)\left(t-t_{0}\right)^{2}
$$

for $0 \leq t_{0}, t<T$. The function $t \rightarrow p_{k}(t, \cdot)$ from $\mathbb{R}_{\geq 0}$ to $\ell_{1}\left(\mathbb{N}_{0}^{d}\right)$ has therefore at the point $t_{0}$ the derivative

$$
\lim _{t \rightarrow t_{0}} \frac{p_{k}(t, \cdot)-p_{k}\left(t_{0}, \cdot\right)}{t-t_{0}}=p_{k+1}\left(t_{0}, \cdot\right) .
$$

This holds also in the polynomially weighted $\ell_{1}$-spaces as can be shown simply adding the corresponding weight factor in the sums. Since $T>0$ and $0 \leq t_{0}<T$ were arbitrary, the proposition follows by induction on $k$.

Let us reformulate this in terms of semigroup theory. We start from assumptions as in Theorem 4.6. Let

$$
E(t): \ell_{1}\left(\mathbb{N}_{0}^{d}\right) \rightarrow \ell_{1}\left(\mathbb{N}_{0}^{d}\right): p(0, \cdot) \rightarrow p(t, \cdot), \quad t \geq 0,
$$

be the operators that assign the solution (4.9) of the equation (4.13) to an initial value $p(0, \cdot)$ in $\ell_{1}\left(\mathbb{N}_{0}^{d}\right)$. As the coefficients of this equation do not depend on time, these operators have the semigroup property

$$
E(s+t)=E(s) E(t), \quad s, t \geq 0 .
$$

The norms of the operators $E(t)$ are by Theorem 4.3 less than or equal 1. As follows from Theorem 4.7,

$$
\lim _{t \rightarrow 0+} E(t) p(0, \cdot)=p(0, \cdot)
$$

for all initial values $p(0, \cdot)$ with finite support. As these are dense in $\ell_{1}\left(\mathbb{N}_{0}^{d}\right),(4.19)$ holds therewith for arbitrary initial values in $\ell_{1}\left(\mathbb{N}_{0}^{d}\right)$. The $E(t)$ thus form a strongly continuous semigroup. The domain of its generator contains by Theorem 4.7 all initial values with finite support. Theorem 4.7 summarizes its regularity properties.

\section{Two COUNTEREXAMPLES}

The question arises whether the basic estimate from Theorem 4.4 is sharp and whether the weighted $\ell_{1}$-norms (4.12) can actually grow exponentially in time. This time the answer is yes, as the example of the equation

$$
\partial_{t} p(t, x)=x p(t, x-1)-(x+1) p(t, x), \quad x=0,1,2, \ldots,
$$

with initial values $p(0, x)=\delta_{x, 0}$ shows. The solution of this initial value problem is unique and given by

$$
p(t, x)=\mathrm{e}^{-t}\left(1-\mathrm{e}^{-t}\right)^{x} .
$$

To calculate its norms (4.12), or in the language of probability theory the moments of these geometric distributions, we first observe that for all real numbers $q$ of absolute value less than one and all natural numbers $m$

$$
\sum_{k=0}^{\infty} k^{m} q^{k}=\frac{q}{1-q} \sum_{k=0}^{\infty}\left((k+1)^{m}-k^{m}\right) q^{k}=\frac{q}{1-q} \sum_{r=0}^{m-1}\left(\begin{array}{c}
m \\
r
\end{array}\right) \sum_{k=0}^{\infty} k^{r} q^{k}
$$

holds. This leads to the representation

$$
\sum_{k=0}^{\infty} k^{m} q^{k}=\frac{1}{(1-q)^{m+1}} P_{m}(q)
$$


where the polynomials $P_{m}(q)$ of degree $m$ are recursively defined by $P_{0}(q)=1$ and

$$
P_{m}(q)=\sum_{r=0}^{m-1}\left(\begin{array}{c}
m \\
r
\end{array}\right)(1-q)^{m-1-r} q P_{r}(q)
$$

for the subsequent $m$. The moments of the distribution (5.2) are thus

$$
\sum_{x=0}^{\infty} x^{m} p(t, x)=P_{m}\left(1-\mathrm{e}^{-t}\right) \mathrm{e}^{m t} .
$$

Since $p(t, x) \rightarrow 0$ for each single $x$ as $t$ goes to infinity, the only possible candidate for a long-time $\ell_{1}$-limit is the zero solution. The existence of this limit contradicts, however, the fact that for all $t$

$$
\sum_{x=0}^{\infty} p(t, x)=1
$$

This observation sheds light on the possible long-time behavior of the solutions of such equations. In the present case the solution simply fades away at infinity. As $P_{m}(1) \neq 0$, more precisely $P_{m}(1)=m$ !, the higher-order moments of the solution grow exponentially in time, asymptotically like $\sim \mathrm{e}^{m t}$. This is essentially the growth that is predicted by Theorem 4.4. Let $\gamma(x)=\max (1, x)^{m}$ be the weight function (1.13). Then

$$
\lim _{x \rightarrow \infty} \frac{(x+1)(\gamma(x+1)-\gamma(x))}{\gamma(x)}=m,
$$

which establishes a direct connection between the growth rate observed here and our central assumption (1.9), respectively the function (1.10) from Theorem 4.4. It is interesting to compare (5.3) with the corresponding quantities for the finite state projections of the given equation on the set of nonnegative integers less than or equal $n$. These finite state projections coincide at the points $x=0,1, \ldots, n$ under consideration with the solution (5.2) of the original problem. Its moments are therefore the partial sums of the infinite series (5.3). These partial sums tend exponentially to zero as $t$ goes to infinity. This effect does not depend on the choice of the initial values, as one recognizes rewriting the corresponding finite system of differential equation in matrix form. The eigenvalues of its coefficient matrix are $\lambda_{k}=-k, k=1, \ldots, n+1$.

Another question is whether growth conditions on the coefficient functions of the given or similar kind are really necessary to have this sort of regularity of the chemical master equation. The example of the equation

$$
\partial_{t} p(t, x)=x^{2} p(t, x-1)-(x+1)^{2} p(t, x)
$$

on the one-dimensional set $x=0,1,2, \ldots$ of integers with initial values $p(0,0)=1$ and $p(0, x)=0$ for $x \geq 1$ shows that one can in general not expect that the norms (4.12) remain bounded for all times if this holds at the initial time. The solutions of this initial value problem can, starting from the solution $p(t, 0)=\mathrm{e}^{-t}$ of

$$
\partial_{t} p(t, 0)=-p(t, 0)
$$

be calculated recursively via the variations of constants formula

$$
p(t, x)=x^{2} \int_{0}^{t} \mathrm{e}^{-(x+1)^{2}(t-s)} p(s, x-1) \mathrm{d} s,
$$

by means of which one obtains by induction on $x$ the lower estimate

$$
p(t, x) \geq \frac{2}{(x+1)(x+2)}\left(\mathrm{e}^{-t}-\frac{4 x}{x+3} \mathrm{e}^{-4 t}\right)
$$

for the solution. For $t \geq \ln (8) / 3$ therefore

$$
p(t, x) \geq \frac{\mathrm{e}^{-t}}{(x+1)(x+2)} .
$$

This means that even the first order moment does not remain finite when time proceeds. 


\section{The Fourier transforms of THE Minimal SOLUTIONS}

It is instructive to change the perspective and to regard the values $p(t, x), x \in \Omega$, of the given nonnegative minimal solutions of the chemical master equation on a subset $\Omega$ of $\mathbb{N}_{0}^{d}$ as coefficients of the Fourier series

$$
\widehat{p}(t, \omega)=\sum_{x \in \Omega} p(t, x) \mathrm{e}^{\mathrm{i} \omega \cdot x}
$$

As with summable initial values the estimate (4.10) holds, this Fourier series converges uniformly for all given times $t \geq 0$ and defines a function that is continuous in $\omega \in \mathbb{R}^{d}$. Estimates like (4.11) for weighted $\ell_{1}$-norms of the solutions translate into statements on the smoothness of the Fourier transforms (6.1):

Theorem 6.1. Assume that for all indices $r$ for which $\left\langle\nu_{r}\right\rangle>0$ the coefficient functions $a_{r}(t, x)$ grow at most linearly in $\langle x\rangle$, that is, satisfy an estimate (1.14). The Fourier transforms (6.1) of the solutions of the chemical master equation (1.1), (1.2) on the state space $\Omega \subseteq \mathbb{N}_{0}^{d}$ for initial values for which the sum

$$
\sum_{x \in \Omega}\langle x\rangle^{m}|p(0, x)|
$$

$m$ a natural number, remains finite are then m-times continuously differentiable functions of $\omega$.

Proof. As discussed in the introduction, the weight function (1.13) satisfies under the given condition to the coefficient functions an estimate (1.9). This implies by Theorem 4.5 that

$$
\sum_{x \in \Omega}\langle x\rangle^{m}|p(t, x)|
$$

remains finite for all times $t \geq 0$. That means that for each given $t$ and every multi-index $\alpha=\left(\alpha_{1}, \ldots, \alpha_{d}\right)$ of nonnegative integers of order $\alpha_{1}+\ldots+\alpha_{d} \leq m$ the partial sums of the Fourier series

$$
\sum_{x \in \Omega}(\mathrm{i} x)^{\alpha} p(t, x) \mathrm{e}^{\mathrm{i} \omega \cdot x}
$$

considered as functions of $\omega$, converge uniformly to a continuous limit function, the corresponding partial derivative of the Fourier transform (6.1) with respect to the variable $\omega$.

Under the given growth condition to the coefficient functions and for corresponding initial values, the Fourier transforms (6.1) of the solutions are therefore infinitely differentiable functions of $\omega$ and their partial derivatives are bounded by the expressions (4.12). Since smooth periodic functions can be approximated in many different ways, observations of this kind may be helpful in the construction and analysis of numerical methods.

\section{The Minimal SOlutions AS PROBABILITy DENSities}

The value $p(t, x)$ represents in chemical kinetics the probability that there are $x_{i}$ copies of the molecule $i$ at time $t$, that is, the solution should behave like a probability density. That means that the sum

$$
\sum_{x \in \Omega} p(t, x)
$$

should be constant in time and take the value 1 for all $t$. This can indeed be shown for the minimal solutions on the full set $\Omega=\mathbb{N}_{0}^{d}$ under a condition on the coefficient functions that is satisfied in chemical kinetics. The main technical problem is that the infinite sum (7.1) cannot simply be differentiated with respect to $t$. 
Lemma 7.1. Assume that $a_{r}(t, x)$ takes the value zero at all $x$ for which $x+\nu_{r}$ possesses at least one negative component. For every minimal solution $p(t, x)$ for summable nonnegative initial values on the set $\Omega=\mathbb{N}_{0}^{d}$ then

$$
\sum_{x \in \Omega} p(t, x)=\sum_{x \in \Omega} p(0, x)
$$

holds for all times $t \geq 0$, on condition that the sums

$$
\sum_{x \in \Omega} a_{r}(t, x) p(t, x)
$$

remain uniformly bounded on finite time intervals.

Proof. Let $\chi$ be the characteristic function of an arbitrary finite subset of $\Omega=\mathbb{N}_{0}^{d}$. Then by (1.1)

$$
\frac{\mathrm{d}}{\mathrm{d} t} \sum_{x \in \mathbb{Z}^{d}} \chi(x) p(t, x)=\sum_{r=1}^{R}\left(\sum_{x \in \mathbb{Z}^{d}} \chi(x) a_{r}\left(t, x-\nu_{r}\right) p\left(t, x-\nu_{r}\right)-\sum_{x \in \mathbb{Z}^{d}} \chi(x) a_{r}(t, x) p(t, x)\right) .
$$

As it extends over the whole $\mathbb{Z}^{d}$, we can shift the arguments in the first of the two inner sums by $\nu_{r}$. This yields

$$
\frac{\mathrm{d}}{\mathrm{d} t} \sum_{x \in \mathbb{Z}^{d}} \chi(x) p(t, x)=\sum_{r=1}^{R} \sum_{x \in \mathbb{Z}^{d}}\left(\chi\left(x+\nu_{r}\right)-\chi(x)\right) a_{r}(t, x) p(t, x) .
$$

The key observation is that one can restrict the inner sum on the right hand side of this equation to those points $x$ for which both $x$ itself and $x+\nu_{r}$ are contained in $\mathbb{N}_{0}^{d}$. The reason is that $p(t, x)$ vanishes for all $x$ outside $\mathbb{N}_{0}^{d}$ and $a_{r}(t, x)$ by assumption for all $x$ for which $x+\nu_{r}$ is not contained in $\mathbb{N}_{0}^{d}$. Integrating this equation,

$$
\sum_{x \in \mathbb{Z}^{d}} \chi(x) p(t, x)-\sum_{x \in \mathbb{Z}^{d}} \chi(x) p(0, x)=\sum_{r=1}^{R} \int_{0}^{t} \sum_{x \in \mathbb{Z}^{d}} \delta_{r}(x) a_{r}(s, x) p(s, x) \mathrm{d} s
$$

follows, where $\delta_{r}(x)=\chi\left(x+\nu_{r}\right)-\chi(x)$ if both $x$ and $x+\nu_{r}$ are contained in $\mathbb{N}_{0}^{d}$ and $\delta_{r}(x)=0$ otherwise. We insert for $\chi$ now the functions $\chi_{n}$ with values $\chi_{n}(x)=1$ for the $x \in \mathbb{N}_{0}^{d}$ with $\langle x\rangle<n$ and $\chi_{n}(x)=0$ for all other $x$. The assigned functions $\delta_{r, n}$ take then a value $\delta_{r, n}(x) \neq 0$, if and only if $x$ and $x+\nu_{r}$ in $\mathbb{N}_{0}^{d}$ and

$$
n \leq\langle x\rangle\left\langle n-\left\langle\nu_{r}\right\rangle \text { if }\left\langle\nu_{r}\right\rangle<0, n-\left\langle\nu_{r}\right\rangle \leq\langle x\rangle<n \text { if }\left\langle\nu_{r}\right\rangle>0 .\right.
$$

Under the given assumption on the sums (7.3), the left hand side of the inequality

$$
\left|\sum_{x \in \mathbb{Z}^{d}} \delta_{r, n}(x) a_{r}(t, x) p(t, x)\right| \leq \sum_{x \in \mathbb{Z}^{d}} a_{r}(t, x) p(t, x)
$$

tends therefore to zero as $n$ goes to infinity and the dominated convergence theorem yields

$$
\lim _{n \rightarrow \infty} \sum_{r=1}^{R} \int_{0}^{t} \sum_{x \in \mathbb{Z}^{d}} \delta_{r, n}(x) a_{r}(s, x) p(s, x) \mathrm{d} s=0 .
$$

Since the left hand side of the equation above tends at the same time to the difference of the values (7.2), this proves the proposition and shows that the values $p(t, x)$ sum up at all times $t$ to the same value.

If one excludes again reactions between two or more given and further external molecules that add mass to the system, the assumption from Lemma 7.1 on the uniform boundedness of the sums (7.3) is no longer needed. 
Theorem 7.2. Let the coefficient functions $a_{r}(t, x)$ be polynomially bounded in $x$, let them grow at most linearly in $x$ in the sense of (1.14) for all indices $r$ for which $\left\langle\nu_{r}\right\rangle>0$, and let $a_{r}(t, x)=0$ for all $x$ for which $x+\nu_{r}$ possesses at least one negative component. For the given minimal nonnegative solutions on $\Omega=\mathbb{N}_{0}^{d}$ then

$$
\sum_{x \in \Omega} p(t, x)=\sum_{x \in \Omega} p(0, x)
$$

holds for all times $t \geq 0$, summable nonnegative initial values provided.

Proof. We assume first that the initial values $p(0, x)$ vanish for all $x$ except for finitely many. We know from the discussion in the previous section, that is, from the boundedness of the expressions (4.12), that under the given assumptions the sums (7.3) then remain finite and bounded on finite time intervals. As just shown, this proves (7.4) under the given restriction to the initial values. To treat the general case, we start from the nonnegative minimal solutions $p_{n}(t, x)$ on $\mathbb{N}_{0}^{d}$ for the initial values $p_{n}(0, x)=p(0, x)$ if $\langle x\rangle<n$ and $p_{n}(0, x)=0$ otherwise. Because of the contractivity of the equation then

$$
\lim _{n \rightarrow \infty} \sum_{x \in \Omega} p_{n}(t, x)=\sum_{x \in \Omega} p(t, x)
$$

for all $t \geq 0$, even uniformly in time. Since (7.4) holds for the functions $p_{n}(t, x)$, this proves the proposition.

A related result has been proved in [23] on the basis of semigroup techniques.

\section{ERROR ESTIMATES FOR THE FINITE STATE PROJECTIONS}

By construction of the solution $p(t, x)$ for given summable nonnegative initial values on the full set $\mathbb{N}_{0}^{d}$,

$$
0 \leq p(\Omega ; t, x) \leq p(t, x)
$$

holds for all of its finite state projections $p(\Omega ; t, x), \Omega$ a finite subset of $\mathbb{N}_{0}^{d}$. The finite state projections $p(\Omega ; t, x)$ converge pointwise monotonously from below to $p(t, x)$ when $\Omega$ increases and approaches $\mathbb{N}_{0}^{d}$. Assuming that the sum (7.1) remains as in Theorem 7.2 constant in time, one gets the computationally accessible representation

$$
\sum_{x \in \mathbb{N}_{0}^{d}}(p(t, x)-p(\Omega ; t, x))=\sum_{x \in \mathbb{N}_{0}^{d}} p(0, x)-\sum_{x \in \Omega} p(\Omega ; t, x)
$$

of the $\ell_{1}$-norm of the error between the unknown nonnegative solution $p(t, x)$ and any of its finite state projections $p(\Omega ; t, x)$. The right hand side of this equation can be kept under control and the state space $\Omega$ if necessary correspondingly adapted and enlarged; see [20] and [22] for details on such procedures. We are in this section, however, not interested in such a posteriori error estimates but in a priori estimates yielding information on the speed of convergence. Such estimates are based on information on the behavior of the solution $p(t, x)$ at points $x$ far away from the origin as provided by Theorem 4.4 or Theorem 4.5. Our starting point is

Lemma 8.1. Let $p(t, x)$ be the given minimal nonnegative solution of the chemical master equation (1.1), (1.2) on the set $\mathbb{N}_{0}^{d}$ and let $p(\Omega ; t, x)$ be the approximation of $p(t, x)$ that takes the same initial values as $p(t, x)$ on the finite subset $\Omega$ of $\mathbb{N}_{0}^{d}$, that vanishes outside $\Omega$, and that solves the equation (1.1) for all $x \in \Omega$. Then

$$
\sum_{x \notin \Omega} p(t, x) \leq \sum_{x \in \mathbb{N}_{0}^{d}}(p(t, x)-p(\Omega ; t, x)) \leq \sum_{x \notin \Omega} p(t, x)+\int_{0}^{t} \sum_{r=1}^{R} \sum_{x \in \Omega(r)} a_{r}(s, x) p(\Omega ; s, x) \mathrm{d} s,
$$

where $\Omega(r)$ denotes the set of all $x \in \Omega$ for which $x+\nu_{r}$ is located outside $\Omega$ but still in $\mathbb{N}_{0}^{d}$. 
Proof. The first inequality follows from (8.1) and the fact that $p(\Omega ; t, x)$ vanishes outside $\Omega$. To prove the other inequality, let $\Omega^{\prime}$ be an arbitrary finite subset of $\mathbb{N}_{0}^{d}$ that covers $\Omega$ and consider the nonnegative difference

$$
e(t, x)=p\left(\Omega^{\prime} ; t, x\right)-p(\Omega ; t, x)
$$

of the corresponding finite state projections. Its $\ell_{1}$-norm satisfies the differential equation

$$
\frac{\mathrm{d}}{\mathrm{d} t} \sum_{x \in \Omega^{\prime}} e(t, x)=\sum_{r=1}^{R} \sum_{x \in \Omega^{\prime}}\left(a_{r}\left(t, x-\nu_{r}\right) e\left(t, x-\nu_{r}\right)-a_{r}(t, x) e(t, x)\right)+\tau(t),
$$

where the remainder is given by the expression

$$
\tau(t)=\sum_{r=1}^{R} \sum_{x \in \Omega^{\prime} \backslash \Omega} a_{r}\left(t, x-\nu_{r}\right) p\left(\Omega ; t, x-\nu_{r}\right) .
$$

As all terms are nonnegative, one gets an upper bound for $\tau(t)$ replacing the inner sum by the sum over all $x$ outside $\Omega$ but still in $\mathbb{N}_{0}^{d}$. Since $p(\Omega ; t, x)=0$ outside $\Omega$, the remainder can therefore be estimated as

$$
\tau(t) \leq \sum_{r=1}^{R} \sum_{x \in \Omega(r)} a_{r}(t, x) p(\Omega ; t, x) .
$$

Since $e(t, x)$ is nonnegative and vanishes outside the finite set $\Omega^{\prime}$,

$$
\sum_{x \in \Omega^{\prime}} a_{r}\left(t, x-\nu_{r}\right) e\left(t, x-\nu_{r}\right) \leq \sum_{x \in \Omega^{\prime}} a_{r}(t, x) e(t, x) .
$$

From the differential equation above and the estimate for the remainder one obtains therefore the estimate

$$
\frac{\mathrm{d}}{\mathrm{d} t} \sum_{x \in \Omega^{\prime}} e(t, x) \leq \sum_{r=1}^{R} \sum_{x \in \Omega(r)} a_{r}(t, x) p(\Omega ; t, x)
$$

for the derivative. Integration yields

$$
\sum_{x \in \mathbb{N}_{0}^{d}}\left(p\left(\Omega^{\prime} ; t, x\right)-p(\Omega ; t, x)\right) \leq \sum_{x \notin \Omega} p\left(\Omega^{\prime} ; 0, x\right)+\int_{0}^{t} \sum_{r=1}^{R} \sum_{x \in \Omega(r)} a_{r}(s, x) p(\Omega ; s, x) \mathrm{d} s
$$

and with that, taking the supremum over all $\Omega^{\prime}$ under consideration, finally the proposition.

We return now to chemical kinetics and our standard case of the polynomial-like weight functions (1.13). We restrict ourselves as before again to the case that for all reactions $r$ for which $\left\langle\nu_{r}\right\rangle>0$ the coefficient functions $a_{r}(t, x)$ grow at most linearly in $\langle x\rangle$, that is, satisfy an estimate (1.14), and exclude with that again reactions between two and more given molecules and further external reactants that add mass to the system. Let

$$
\Omega_{n}=\left\{x \in \mathbb{N}_{0}^{d} \mid\langle x\rangle<n\right\} .
$$

The sets $\Omega_{n}(r)$ from Lemma 8.1 are in this case empty for all reactions $r$ for which $\left\langle\nu_{r}\right\rangle \leq 0$. If $\left\langle\nu_{r}\right\rangle>0$,

$$
\Omega_{n}(r)=\left\{x \in \mathbb{N}_{0}^{d} \mid x+\nu_{r} \in \mathbb{N}_{0}^{d}, n-\left\langle\nu_{r}\right\rangle \leq\langle x\rangle<n\right\} .
$$


If the weighted $\ell_{1}$-norm (4.12) of the solution $p(t, x)$ is finite at time $t=0$ and remains therefore under the given assumptions to the coefficient functions by Theorem 4.5 uniformly bounded on finite time intervals, the terms on the right hand side of (8.3) can be estimated using (1.14) and (8.1). As, for $n>\left\langle\nu_{r}\right\rangle$,

$$
\sum_{x \in \Omega_{n}(r)} a_{r}(s, x) p\left(\Omega_{n} ; s, x\right) \leq \alpha(s) \sum_{x \in \Omega_{n}(r)}\langle x\rangle p(s, x)<\alpha(s)\left(n-\left\langle\nu_{r}\right\rangle\right)^{-m} n \sum_{x \in \Omega_{n}(r)}\langle x\rangle^{m} p(s, x)
$$

for those indices $r$ for which $\left\langle\nu_{r}\right\rangle>0$ and thus $\Omega_{n}(r) \neq \emptyset$,

$$
\sum_{r=1}^{R} \sum_{x \in \Omega_{n}(r)} a_{r}(s, x) p\left(\Omega_{n} ; s, x\right) \leq \frac{1}{n^{m-1}}\left(\sum_{\left\langle\nu_{r}\right\rangle>0}\left(\frac{n}{n-\left\langle\nu_{r}\right\rangle}\right)^{m}\right) \alpha(s) \sum_{x \in \mathbb{N}_{0}^{d}}\langle x\rangle^{m} p(s, x)
$$

for correspondingly large values of $n$. As furthermore

$$
\sum_{x \notin \Omega_{n}} p(t, x) \leq \frac{1}{n^{m}} \sum_{x \in \mathbb{N}_{0}^{d}}\langle x\rangle^{m} p(t, x),
$$

one obtains therefore the following convergence result:

Theorem 8.2. Provided the norm (4.12) of the solution under consideration is finite at the initial time and thus remains uniformly bounded on bounded time intervals, the error tends on every interval $0 \leq t \leq T$ like

$$
\sup _{0 \leq t \leq T} \sum_{x \in \mathbb{N}_{0}^{d}}\left(p(t, x)-p\left(\Omega_{n} ; t, x\right)\right)=\mathcal{O}\left(n^{-m+1}\right)
$$

to zero as $n$ goes to infinity and the finite state spaces (8.4) correspondingly expand.

The size of the constant will in general strongly increase with $m$ and the length of the time interval under consideration but is basically independent of the dimension $d$ of the state space, that is, in chemical kinetics of the number of species involved. The curse of dimensionality is hidden in the choice of the state spaces (8.4). We conclude that, corresponding initial values provided, the finite state projections $p\left(\Omega_{n} ; t, x\right)$ converge superalgebraically to the solution, faster than any power of $1 / n$ tends to zero.

The finite state projections of a solution of a chemical master equation do in general not remain probability densities when time proceeds. It is therefore common practice to modify the equation slightly near the boundary of the given finite domain $\Omega$, replacing the coefficient functions $a_{r}(t, x)$ by their truncated variants

$$
a_{r}(\Omega ; t, x)=\left\{\begin{array}{l}
a_{r}(t, x), \text { if } x+\nu_{r} \in \Omega, \\
0, \text { otherwise. }
\end{array}\right.
$$

The solutions $\widetilde{p}(\Omega ; t, x)$ of the so modified equations (1.1), (1.2) have then again the property that

$$
\sum_{x \in \Omega} \widetilde{p}(\Omega ; t, x)=\sum_{x \in \Omega} \widetilde{p}(\Omega ; 0, x) .
$$

The proof transfers almost verbatim from that of Lemma 7.1, simply replacing the full set $\mathbb{N}_{0}^{d}$ by the given finite set $\Omega$. The only difference is that one can, as $\Omega$ is itself a finite set, directly insert the characteristic function $\chi$ of $\Omega$ there, which makes the detour via a limit process as in the proof of Lemma 7.1 unnecessary. The question is which impact this modification has on the discretization error.

Lemma 8.3. If $\widetilde{p}(\Omega ; 0, x)=p(\Omega ; 0, x)$ for all $x$ in the finite set $\Omega$, for all times $t \geq 0$

$$
p(\Omega ; t, x) \leq \widetilde{p}(\Omega ; t, x) .
$$


Proof. The argumentation follows that in the proof of Lemma 2.1. We restrict ourselves at first again to an arbitrarily chosen finite time interval. Let $\mu$ be an upper bound for the sum (1.4) of the coefficient functions for all $x$ in the finite domain $\Omega$ under consideration and all $t$ in this time interval. Set

$$
e(t, x)=\mathrm{e}^{\mu t}\{\widetilde{p}(\Omega ; t, x)-p(\Omega ; t, x)\} .
$$

Because of $a_{r}\left(\Omega ; t, x-\nu_{r}\right)=a_{r}\left(t, x-\nu_{r}\right)$ for the points $x \in \Omega$, for all $x \in \Omega$ then

$$
\frac{\mathrm{d}}{\mathrm{d} t} e(t, x)=\mu e(t, x)+\sum_{r=1}^{R}\left(a_{r}\left(\Omega ; t, x-\nu_{r}\right) e\left(t, x-\nu_{r}\right)-a_{r}(\Omega ; t, x) e(t, x)\right)+\tau(t, x),
$$

where the remainder is given by the expression

$$
\tau(t, x)=\mathrm{e}^{\mu t} \sum_{r=1}^{R}\left(a_{r}(t, x)-a_{r}(\Omega ; t, x)\right) p(\Omega ; t, x)
$$

and thus nonnegative. This implies by the same arguments as in the proof of Lemma 2.1 that $e(t, x)$ and with that also difference of the functions $\widetilde{p}(\Omega ; t, x)$ and $p(\Omega ; t, x)$ is nonnegative on the given time interval.

To estimate the $\ell_{1}$-distance between the original finite state projection and its modification, we utilize the same condition on the coefficient functions that was needed in Section 6 to prove the conservation property (7.2) and that avoids that the solutions leak from the domain.

Lemma 8.4. Assume that $a_{r}(t, x)=0$ if $x+\nu_{r} \notin \mathbb{N}_{0}^{d}$ and let $\Omega(r)$ as in Lemma 7.1 consist of the $x \in \Omega$ for which $x+\nu_{r}$ is located outside $\Omega$ but still in $\mathbb{N}_{0}^{d}$. If $\widetilde{p}(\Omega ; 0, x)=p(\Omega ; 0, x)$ for all $x \in \Omega$, for all times $t \geq 0$ then

$$
\sum_{x \in \Omega}(\widetilde{p}(\Omega ; t, x)-p(\Omega ; t, x)) \leq \int_{0}^{t} \sum_{r=1}^{R} \sum_{x \in \Omega(r)} a_{r}(s, x) p(\Omega ; s, x) \mathrm{d} s .
$$

Proof. We start again from the by Lemma 8.3 nonnegative difference

$$
e(t, x)=\widetilde{p}(\Omega ; t, x)-p(\Omega ; t, x)
$$

of the modified and the original finite state projection. As in the proof of the previous lemma for all $x \in \Omega$ then

$$
\frac{\mathrm{d}}{\mathrm{d} t} e(t, x)=\sum_{r=1}^{R}\left(a_{r}\left(\Omega ; t, x-\nu_{r}\right) e\left(t, x-\nu_{r}\right)-a_{r}(\Omega ; t, x) e(t, x)\right)+\delta(t, x) p(\Omega ; t, x),
$$

where we have used the abbreviation

$$
\delta(t, x)=\sum_{r=1}^{R}\left(a_{r}(t, x)-a_{r}(\Omega ; t, x)\right)
$$

here. Since $e(t, x)$ is nonnegative and vanishes outside the finite set $\Omega$,

$$
\sum_{x \in \Omega} a_{r}\left(\Omega ; t, x-\nu_{r}\right) e\left(t, x-\nu_{r}\right) \leq \sum_{x \in \Omega} a_{r}(\Omega ; t, x) e(t, x) .
$$

From the differential equation above one obtains therefore the estimate

$$
\frac{\mathrm{d}}{\mathrm{d} t} \sum_{x \in \Omega} e(t, x) \leq \sum_{x \in \Omega} \delta(t, x) p(\Omega ; t, x)
$$


for the derivative of the norm. As $e(0, x)=0$, integration yields

$$
\sum_{x \in \Omega}(\widetilde{p}(\Omega ; t, x)-p(\Omega ; t, x)) \leq \int_{0}^{t} \sum_{r=1}^{R} \sum_{x \in \Omega}\left(a_{r}(s, x)-a_{r}(\Omega ; s, x)\right) p(\Omega ; s, x) \mathrm{d} s .
$$

Using that the difference $a_{r}(s, x)-a_{r}(\Omega ; s, x)$ takes the value $a_{r}(s, x)$ for the elements $x \in \Omega(r)$ and vanishes everywhere else in $\Omega$, the proposition follows.

The additional error introduced by the truncation of the coefficient functions can thus, under an assumption without that the modification (8.10) does not make sense, be estimated by the same terms as the original error. The observed convergence behavior is therefore not affected by the change of the coefficient functions.

\section{REFERENCES}

[1] P. Deuflhard, W. Huisinga, T. Jahnke and M. Wulkow, Adaptive discrete Galerkin methods applied to the chemical master equation. SIAM J. Sci. Comput. 30 (2008) 2990-3011.

[2] S.V. Dolgov and B.N. Khoromskij, Simultaneous state-time approximation of the chemical master equation using tensor product formats. arXiv:1311.3143 (2013).

[3] S. Engblom, Spectral approximation of solutions to the chemical master equation. J. Comput. Appl. Math. 229 (2009) $208-221$.

[4] D.T. Gillespie, A general method for numerically simulating the stochastic time evolution of coupled chemical reactions. $J$. Comput. Phys. 22 (1976) 403-434.

[5] D.T. Gillespie, A rigorous derivation of the chemical master equation. Phys. A 188 (1992) 404-425.

[6] D.T. Gillespie, Stochastic simulation of chemical kinetics. Annu. Rev. Phys. Chem. 58 (2007) 35-55.

[7] M. Hegland, Approximating the solution of the chemical master equation by aggregation. ANZIAM J. 50 (2008) C371-C384.

[8] M. Hegland and J. Garcke, On the numerical solution of the chemical master equation with sums of rank one tensors. ANZIAM J. Electron. Suppl. 52 (2010) C628-C643.

[9] M. Hegland, A. Hellander and P. Lötstedt, Sparse grids and hybrid methods for the chemical master equation. BIT 48 (2008) 265-283.

[10] A. Hellander and P. Lötstedt, Hybrid method for the chemical master equation. J. Comput. Phys. 227 (2007) 100-122.

[11] D.J. Higham, Modeling and simulating chemical reactions. SIAM Rev., 50:347-368, 2008.

[12] S. Ilie, W.H. Enright and K.R. Jackson, Numerical solution of stochastic models of biochemical kinetics. Can. Appl. Math. Q. 17 (2009) 523-554.

[13] T. Jahnke, On reduced models for the chemical master equation. Multiscale Model. Simul. 9 (2011) 1646-1676.

[14] T. Jahnke and W. Huisinga, A dynamical low-rank approach to the chemical master equation. Bull. Math. Biol. 70 (2008) 2283-2302.

[15] T. Jahnke and T. Udrescu, Solving chemical master equations by adaptive wavelet compression. J. Comput. Phys. 229 (2010) $5724-5741$.

[16] V. Kazeev, M. Khammash, M. Nip and Ch. Schwab, Direct solution of the chemical master equation using quantized tensor trains. PLoS Comput. Biol. 10 (2014) e1003359.

[17] W. Ledermann and G.E.H. Reuter, Spectral theory for the differential equations of simple birth and death processes. Phil. Trans. R. Soc. A 246 (1954) 321-369.

[18] M. Martcheva, H.R. Thieme and T. Dhirasakdanon, Kolmogorov's differential equations and positive semigroups on first moment sequence spaces. J. Math. Biol. 53 (2006) 642-671.

[19] S. Menz, J.C. Latorre, C. Schütte and W. Huisinga, Hybrid stochastic-deterministic solution of the chemical master equation. Multiscale Model. Simul. 10 (2012) 1232-1262.

[20] B. Munsky and M. Khammash, The finite state projection algorithm for the solution of the chemical master equation. J. Chem. Phys. 124 (2006) 044104.

[21] G.E.H. Reuter and W. Ledermann, On the differential equations for the transition probabilities of Markov processes with enumerably many states. Proc. Cambridge Philos. Soc. 49 (1953) 247-262.

[22] V. Sunkara and M. Hegland, An optimal finite state projection method. Procedia Comput. Sci. 1 (2012) 1579-1586.

[23] H.R. Thieme and J. Voigt, Stochastic semigroups: their construction by perturbation and approximation, in Positivity IV-theory and applications. Tech. Univ. Dresden, Dresden (2006) 135-146.

[24] T. Udrescu, Numerical methods for the chemical master equation. Doctoral Thesis, Karlsruher Institut für Technologie (2012). 